\title{
BASES CHIMIOSENSORIELLES DU COMPORTEMENT ALIMENTAIRE CHEZ LES POISSONS
}

\author{
Ph. SAGLIO*
}

RESUME

Le comportement alimentaire, indispensable à la survie de l'individu et donc de l'espèce, occupe à ce titre une position de première importance dans la hiérarchie des comportements fondamentaux qui tous en dépendent très étroitement.

Chez les poissons, cette prééminence se trouve illustrée par l'extrême diversité des supports sensoriels impliqués et des expressions comportementales qui leur sont liées.

A la suite d'un certain nombre de mises en évidence neurophysiologiques et éthologiques de l'importance du sens chimique (olfaction, gustation) dans le comportement alimentaire des poissons, de très importants secteurs d'études électrophysiologiques et d'analyses physico-chimiques visant à en déterminer la nature exacte (en-termes de substances actives) se sont développés ces vingt dernières années.

De tous ces travaux dont les plus avancés sont présentés ici, il ressort que les acides aminés de série $L$ plus ou moins associés à d'autres composés de poids moléculaires $<1000$ constituent des composés chimiques jouant un rôle céterminant dans le comportement alimentaire de nombreuses espèces de poissons carnivores.

\section{ABSTRACT}

Feeding behavior, essential to both individual and species survival, must be considered as the first fundamental behavior in so far as all the others closely depend on its occurence. In fishes, this pre-eminent position is well illustrated by the extreme diversity of sensorial paths and behavioral expressions involved. Following neurophysiological and ethological evidences of the importance of chemical sense in feeding behavior of fishes, electrophysiological and chemical analysis, dealing more precisely with the identity of the chemicals involved, have been widely worked out these last two decades.

We present here some studies showing the fact that, in freshwater as in marine carnivorous fish species, amino acids, possibly associated with other low molecular size compounds $(<1000)$, can actively contribute to the effectiveness of food odors.

\section{PRESENTATION}

Le comportement alimentaire, comme tout comportement fondamental, peut être présenté comme séquentiel. Chez les poissons, comme chez la plupart des animaux, les étapes constituant cette séquence sont :

- L'EVEIL au stimulus alimentaire

- L'ORIENTATION ET LA RECHERCHE de l'aliment

- LA PRISE DE NOURRITURE dans la bouche

- SON INGESTION

Bien que liées entre elles, ces étapes subissent, selon les données biologiques de l'espèce et de l'éco-système considéré, un contrôle physico-chimique, et donc sensoriel particulier.

Même chez les espèces qualifiées de visuelles prédominantes (cas des prédateurs chassant à l'affüt comme le brochet) ou encore chez les espèces à chimiosensorialité extrê. mement développée (comme celles appartenant aux familles des Myxinidés, des Situridés et des Anguillidès), il est difficile de soutenir que ce comportement complexe puisse être déterminé par le fonctionnement d'un seul et unique canal sensoriel.

Les expériences de lésions sensorielles (outre les effets préjudiciables sur l'ensemble des expressions comportementales propres à l'espèce) manquent le plus souvent d'objectivité sinon d'exactitude dans la mesure où elles créent une opposition entre deux voies sensorielles, olfactive ou visuelle, à l'exclusion de toute autre. Or, outre la ligne latérale, les bourgeons du goût ou autres organes gustatifs bien différenciés (cirres buccales des siluridés et rayons de la nageoire pectorale des Triglidés: par exemple), le poisson peut

* Laboratoire d'Ecologie des Poissons et d'Aménagement des Pêches, B.P. no 3, Saint-Pée-sur-Nivelle 64310 ASCAIN. 
également présenter une électrosensorialité ou une sensibilité auditive particulière jouant un rôle actif dans le comportement alimentaire. Ceci a été démontré chez de nombreuses espèces (KEENLEYSIDE, 1979).

Ainsi, tant par le nombre et la diversité des voies sensorielles susceptibles d'être recrutées que par la spécificité existant aussi bien dans l'accord entre ces différentes voies que dans la nature des signaux efficaces (variable non seulement au niveau de l'espèce, mais aussi au niveau de l'individu, au cours de son ontogénèse), le comportement alimentaire des Poissons se présente globalement comme possédant un déterminisme sensoriel et une variabilité conséquente des motivations et des expressions, particulièrement remarquable.

Laissant volontairement de côté la description analytique des structures chimiosensorielles impliquées, nous nous attacherons ici ̀̀ présenter les premières conclusions des travaux ayant porté sur le déterminisme chimique du comportement alimentaire des Poissons.

\section{IMPORTANCE DU PHENOMENE}

Le rôle du sens chimique dans le comportement alimentaire des Poissons, encore énigmatique chez les espèces herbivores, a été principalement mis en évidence chez les détritivores, des nécrophages et des carnivores.

Chez les détritivores, poissons, se nourrissant de matières organiques provenant d'organismes morts ou d'excrétions animales, BENSON et MUSCATINE (1974) ont montré que de nombreuses espèces (se rattachant en particulier aux familles des Chætodontidés, des Pomacentridés et des Monacanthidés) sont très attirées par le mucus exsudé en abondance par les polypes lorsqu'un morceau de corail vient à se briser.

Chez les nécrophages, espèces se nourrissant d'organismes morts ou mourants, la découverte de l'aliment se fait très probablement par voie chimique (et peut-être aussi par voie auditive dans le ca's des proies se débattant) dans la mesure où les arganismes morts ou agonisants reposent habituellement sur le fond à des profondeurs où le faible éclairement ou la nature du substrat permet difficilement à la vision de s'exprimer.

Ceci est confirmé par le développement important des voies gustatives et (ou) olfactives de ces espèces benthiques nécrophages. Ainsi, la Myxine (Myxina glutinosa), poisson marin benthique aux yeux rudimentaires, répond à l'odeur de la proie par une remontée active du courant jusqu'à la source de stimulus chimique (chimio-rhéotaxie positive). Une fois arrivée au contact de la proie, la myxine se fixe et pénètre les téguments grâce à ses dents linguales (STRAHAN, 1963)

Outre ces deux catégories, c'est parmi le groupe dominant par le nombre d'espèces des poissons carnivores que le support chimiosensoriel du comportement alimentaire est le mieux connu.

Précédant les nombreuses études menées au laboratoire, un certain nombre d'observations de terrain rendent compte de l'intervention du sens chimique dans la détermination, la capture et l'ingestion de la proie.

Chez les Catostomidés et chez certains Cichlidés d'eau douce (Geophagus et Lethrinops), le poisson, après prélèvement buccal de substrat, en rejette la plus grande partie pour ne garder que les matières organiques, larves d'invertébrés ou vers. Le choix de l'endroit de orélèvement du substrat repose ici selon toute vraisemblance sur le sens chimique de ces poissons.

Chez de nombreuses espèces de Sélaciens, outre la capacité de percevoir les signaux acoustiques ou le champ bio-électrique émis par leurs proies, il existe une chimiosensorialité très développée.

HOBSON (1963) et TESTER (1963) ont montré chez Musculatus canis et Carcharhinus menisorrah que la présence de proies produisait une augmentation de l'activité générale suivie d'une nage d'approche à contre courant.

\section{LE SUPPORT CHIMIQUE}

Le protocole suivi par les auteurs cherchant à déterminer les bases chimiques du comportement alimentaire d'une espèce de poisson suit assez invariablement les grandes lignes suivantes:

1 - Elevage au laboratoire

2 - Tests comportementaux (olfactométrie) et électrophysiologiques des différents organismes ou tissus animaux sous leur forme brute ou sous la forme d'extraits aqueux.

3 - Analyse physico-chimique de l"extrait actif.

4 - Nouveaux tests comportementaux et électrophysiologiques destinés à contrôler l'activité des fractions isolées et, dans les meilleurs des cas, des composés identifiés après analyse.

5 - Contrôle d'activité sur le terrain. 
Toutefois, ce plan d'action, identique par la structure à celui appliqué à l'étude de toute communication chimique, ne peut être mis en cuvre de façon optimale que par des équipes constituées, très rares à l'heure actuelle, et il n'existe pratiquement pas d'exemple d'espèces chez lesquelles ces cinq étapes ont pu être bouclées.

Malgré ceci. les études menées de par le monde ces deux dernières décennies ont permis d'isoler un certain nombre d'extraits et même des composés actifs sur le comportement alimentaire de Poissons carnivores appartenant entre autres aux familles des Pétromyzonidés (KLEEREKOPER et MOGENSEN, 1963), des Sélaciens (HODGSON et al., 1967; KLEEREKOPER, 1967; MATHEWSON et HODGSON, 1972), des Siluridés (FUJIYA et BARDACH, 1966; BARDACH et al.. 1967), des Salmonidés (McBRIDE et al., 1962). des Anguillidés (HASHIMOTO et al., 1968), des Gadidés (PAWSON, 1977), des Pleuronectidés (SUTTERLIN, 1975), des Sparidés (CARR et CHANEY, 1975) et des Pomadasyidés (CARR, 1976).

Chez certaines espèces que I'on pourrait qualifier d'euryphages, telles que la Capucette (Hepsitia stipes, STEVEN, 1959) ou l'anguille japonaise (Anguilla japonica, HASHIMOTO et al., 1968), le comportement de recherche alimentaire peut être provoqué par une variété d'extraits d'origine animale sans relation obligatoire avec les ressources disponibles dans le milieu naturel, et donc, dans ce cas, sans nécessité d'un conditionnement préalable.

Chez l'anguille, les expériences de conditionnement alimentaire menées par ces auteurs à partir d'extraits de foie de bcuf, de vers oligochètes ou de crevettes, ne faisaient apparaître aucune préférence.

De même, chez la lamproie marine (Petromyzon marinus) KLEEREKOPER et MOGENSEN (1963) montraient que la réponse alimentaire (augmentation de l'activité locomotrice) provoquée par l'introduction d'eau ayant contenu préalablement des truites. se manifestait également chez des animaux n'ayant jamais été soumis à l'odeur de cette proie.

A l'inverse, chez des smolts de saumons sockeye (Oncorhynchus nerka) McBRIDE et al. (1962) n'obtenaient de réponses (<éclatement - du banc, augmentation de la vitesse de nage et passage dans une zone éclairée) qu'avec les extraits auxquels ces poissons avaient subi un conditionnement alimentaire antérieur d'au moins dix. jours.

Ainsi, le zooplancton et les calmars testés, bien que constituant le régime alimentaire du Sockeye adulte, ne produisaient aucune activité chez les smolts non conditionnés, tandis que de l'extrait de foie de bœuf, évidemment non représentatif du régime de cette espèce, était actif moyennant ce conditionnement préalable.

L'inactivité des extraits lors de leur première présentation doit être également mise en relation'avec le fait qu'aucun de ceux-ci ne reflétait les possibilités alimentaires du milieu originel de ces poissons, au stade auquel ils ont été pêchés.

D'après ces travaux, le Sockeye se présenterait donc comme une espèce sténophage dont les exigences alimentaires seraient toutefois susceptibles de conditionnement et de variation qualitative au cours de l'ontogénèse.

Après analyse de ces différents extraits d'origine animale, il ressort que les substances actives, de nature non volatile et de poids moléculaire inférieur à 1000 , sont constituées essentiellement d'acides aminés parmi lesquels, et par ordre d'importance, l'arginine, l'alanine, la glycine, l'acide glutamique, la méthionine, la phènylalanine, l'histidine et la thréonine, tous de série $\mathrm{L}$, occupent une place dominante. Le seuil minimum de concentration d'un acide aminé produisant une réponse est de l'ordre de $10^{-6} \mathrm{M}$ pour l'aspect comportemental et. jusqu'à $10^{-9} \mathrm{M}$ pour l'aspect électrophysiologique.

Si l'on ne peut qu'apprécier les très nombreux enregistrements électrophysiologiques réalisés ces dernières années à partir des voies olfactives ou gustatives de poissons d'espèces variées et les informations recueillies par cette méthode concernant le caractère excitateur de ces composés sur le système chimiosensoriel de ces animaux (CAPRIO, 1974, 1977, 1978; GOH et TAMURA, 1978; GOH et al., 1979; HARA, 1973, 1976, 1977; HARA et al., 1973; SUTTERLIN et SUTTERLIN, 1971; SUZUKI et TUCKER; 1971), il est regrettable que ceux-ci n'aient été que si rarement suivis d'observations comportementales.

On en arrive aujourd'hui à une situation de recherche où l'on en connait beaucoup plus sur les phénomènes bioélectriques existant au niveau de structures neurosensorielles telles que l'épithélium olfactif, le bulbe olfactif ou le barbillon gustatif que sur la nature des comportements en relation avec de tels phénomènes. Bien que ce déséquilibre, en opposition avec tout développement ou application de ce domaine d'étude, ne cesse de se confirmer, il existe quelques travaux comme ceux de SUTTERLIN (1975), de CARR et CHANEY (1975), de CARR et al., (1975), de CARR (1976) et de PAWSON (1977) qui constituent de remarquables exceptions.

Chez la Plie rouge (Pseudopleuronectes americanus), le Choquemort (Fundulus heteroclitus) et la Capucette (Menidia menidia), poissons de I'Atlantique Nord, SUTTERLIN est parvenu à montrer dans le milieu naturel, grâce à l'utilisation combinée de trappes à pois- 
sons et de techniques vidéo très sophistiquées, qu'il était possible d'attirer sélectivement par moyen chimique chacune de ces espèces (Tableaux 1 et 2).

Tableau 1 : Caractère attractif de différents composés sur la plie rouge (Pseudopleuronectes americanus), testés à une concentration (niveau tube d'émission) de $10^{-3} \mathrm{M}$, (d'après SUTTERLIN 1975).

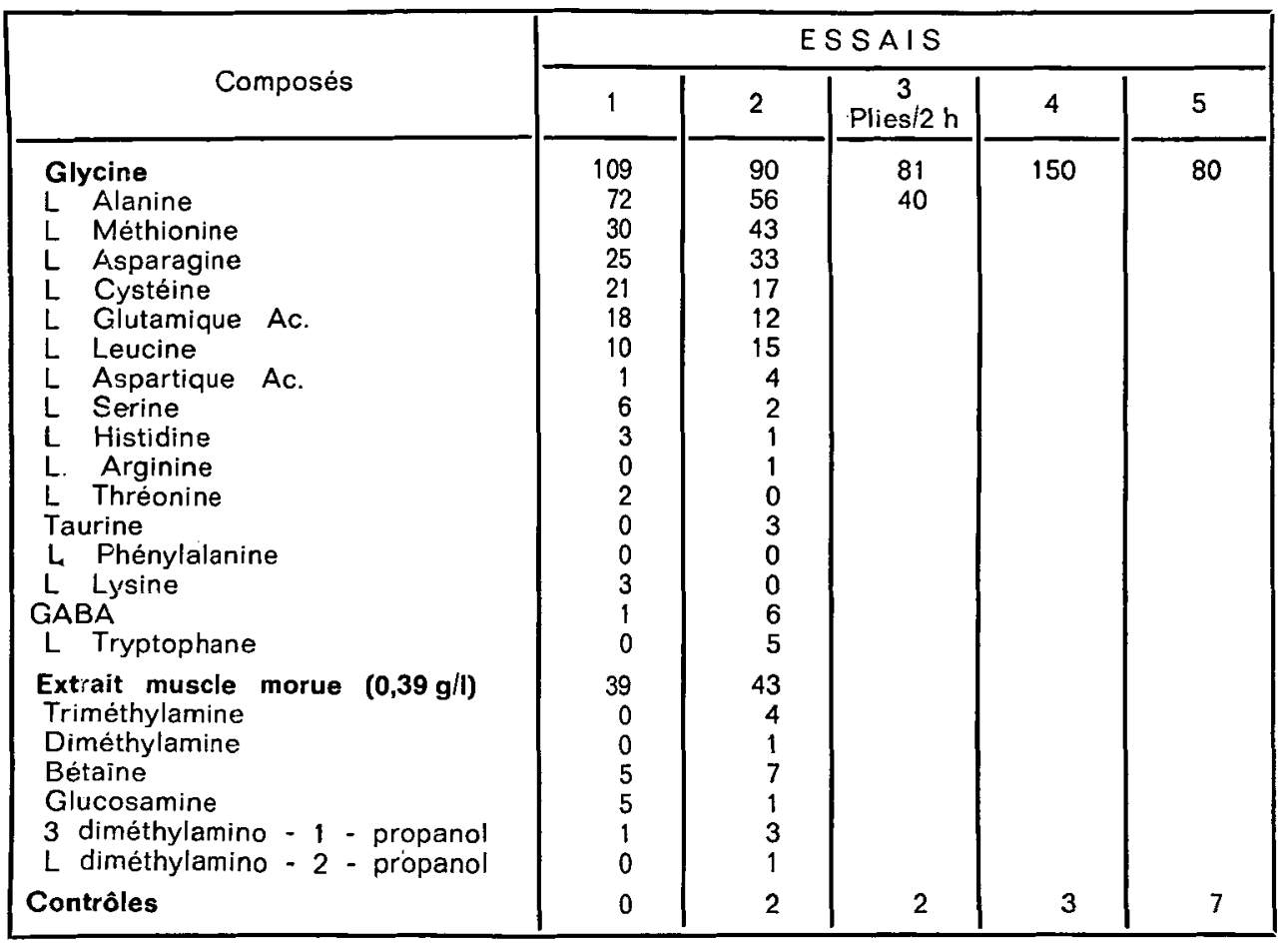

Tableau 2 : Caractère attractif de différents composés sur le Choquemort (Fundulus heteroclitus) et la Capucette (Menidia menidia) à une concentration (niveau tube d'émission) de $10^{-4} \mathrm{M}$.

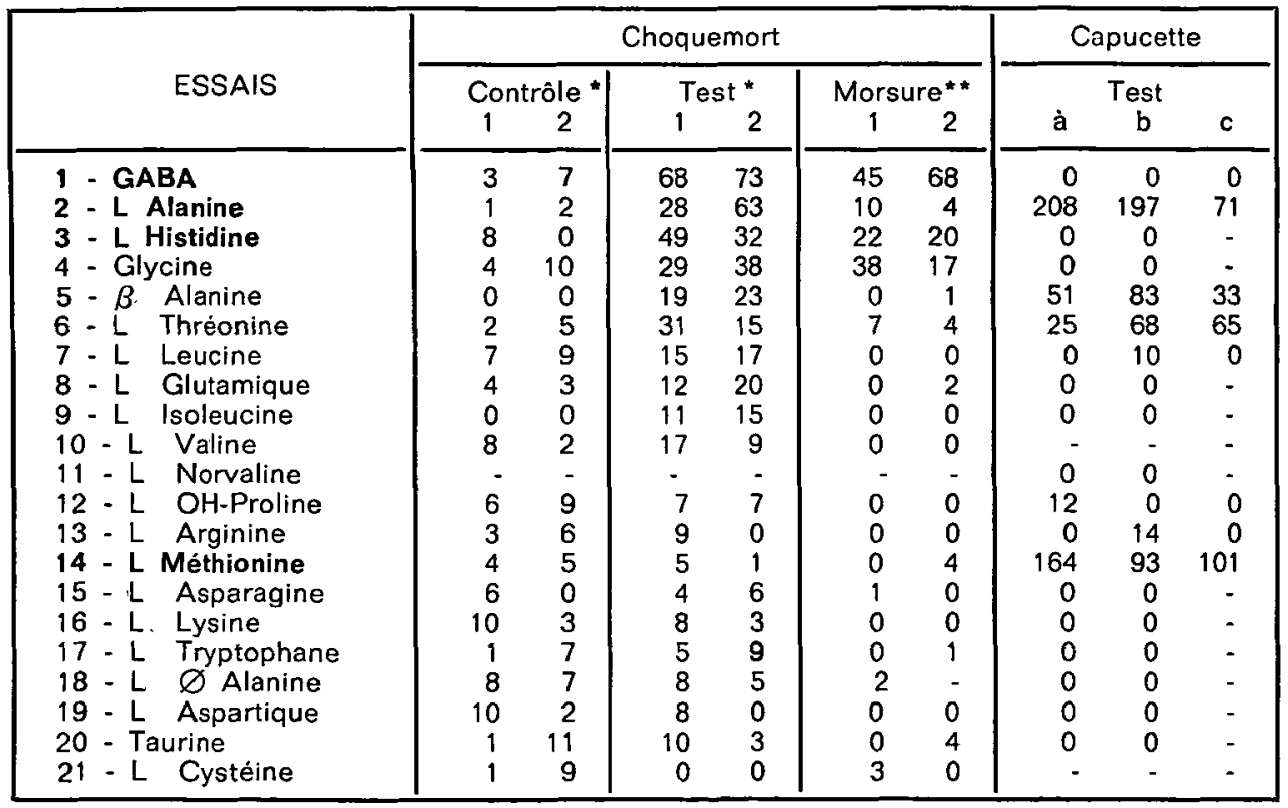

- Nombre moyen de poissons pour 10 répétitions

- Nombre de poissons présentant une réponse de morsure par 10 minutes 
II apparait à l'examen de ces tableaux certains groupes de composés différenciables par le degrè de spécificité de leur caractère attractif défini par rapport aux contrôles: - Des composés très attractifs à haute spécificité tel le GABA (Ac. $\gamma$ amino-butyrique) sur le Choquemort.

- Des composés très attractifs à spécificitè moyenne telles la Glycine sur la Plie rouge, la $L$. Alanine ou la L. Methionine. sur la Capucette.

- Des composès d'attractivité moyenne à haute spécificité, telle la L. Histidine sur le Choquemort.

- Dautres composés possedant une attractivité insuffisante pour permettre d'en apprécier le caractère spécifique.

Ayant noté l'efficacité de l'Arenicole (Arenicola marina) en tant que leurre sélectif pour la capture de deux Gadidés (Merlangus merlangus et Gadus morhua) présents dans le sud-est des côtes anglaises, PAWSON entreprit l'étude analytique de ce phénomène. Après réalisation d'extraits d'arénicoles, chromatographie et détermination au moyen du test olfactométrique de la fraction active, l'auteur én arriva à la conclusion que celle-ci contenait de la Glycine, de la Sérine, de l'Alanine, de l'acide Glutamique, de la Valins, de la Leucine et de la Thréonine. De tous ces acides aminés, seuls la Glycine et l'Alanine étaient capables de produire des réponses alimentaires significatives lorśqu'ils étaient testés individuellement.

Bien que les Lipides et les Hydrates de carbone soient inactifs sur le comportement alimentaire de ces poissons carnivores, d'autres composés azotés tel que la Bétaine $\left[\left(\mathrm{CH}_{3}\right)_{3}-\mathrm{N}^{+}-\mathrm{CH}_{2}-\mathrm{COO}^{-}\right]$peuvent entrer pour une part importante dans l'activité des $\in \mathrm{X}$ traits analysés.

Chez deux espèces de poissons d'estuaires du sud-est des Etats-Unis, Lagodon rhómboides (Sparidé) et Orthopristis Chrysopterus (Pomadasyidé), CARR a montré après analyse d'extraits de crevettes (Penaeus duorarum) que la concentration de ce composé par rapport aux 20 acides aminés isolés représentait à lui seul le tiers de la concentration totale en acides aminés libres et possédait une activité sur le comportement alimentaire égale à $25 \%$ de celle de l'extrait brut pour Lagodon rhomboïdes et à $40 \%$ pour Orthopristis chrysopterus.

\section{CONCLUSIONS}

Considérant ces données et le fait qu'aucun acide aminé, pris isolément́, n'est aussi actif qu'en mélange, le comportement alimentaire des poissons carnivores pourrait dépendre de l'intégration chimiosensorielle d'un système à plusieurs composants dont lunité élémentaire est l'acide aminé.

La spécificité serait alors assurée par des variations qualitatives et quantitatives de ces composés auxquelles se joindraient par ailleurs la présence active d'autres composés de structure très proche et de poids moléculaires voisins de ceux-ci dont la Bétaíne constituerait un exemple.

Vu leurs propriétés physico-chimiques (polarité et faible volatilité en particulier) il n'est guère surprenant que des acides aminés soient aussi actifs sur le comportement alimentaire d'animaux aquatiques chez lesquels ils peuvent agir comme médiateurs chimiques à distance. Cette activité n'est guère restreinte aux poissons et a été également mise en évidence chez de nombreux invertébrés aquatiques (LINDSTEDT, 1970). Toutefois, alors que des protéines peuvent également stimuler le comportement alimentaire de plusieurs espèces d'invertébrés marins (CARR et GURIN, 1975), les observations comportementales menées chez les poissons ont montré jusqu'à présent que l'activité n'était obtenue que pour des composés de poids moléculaires très inférieurs.

Bien que ce domaine de recherches nécessite une interdisciplinarité assez lourde à mettre en œuvre, il est, d'un certain point de vue, tout à fait encourageant de noter qu'il existe déjà des composés susceptibles de produire l'attraction alimentaire d'espèces variées de poissons.

S'il est vrai que l'action éco-éthologique de ces composés demeure encore un mystère sur lequel il convient de se pencher tout particulièrement (nature de la réponse prise dans sa totalité, concentration optimale, action éventuelle sur les autres organismes présents dans le milieu propre à l'espèce, importance de la qualité physico-chimique du milieu), nous pouvons raisonnablement penser, vu les avantages évidents qui pourraient résulter de leur judicieuse utilisation, que ce secteur continuera de s'étendre dans les prochaines années.

\section{BIBLIOGRAPHIE}

BARDACH J.E., TODD J.H., CRICKMER R., 1967. Orientation by taste in fish of the genus ictalurus. Science, $155: 1276-1278$. 
BENSON A.A., MUSCATINE L., 1974. Wax in coral mucus : energy transfer from corals to reef fishes. Limnol. Oceanogr., $19: 810-814$.

CAPRIO J., 1975. High sensitivity of catfish taste receptors to amino acids. Comp. biochem. Physiol, 52 A : 247 - 251.

CAPRIO J., 1977. Electrophysiological distinctions between the taste and smell of amino-acids in catfish. Nature, 266, $5605: 850-851$.

CAPRIO J., 1978. Olfaction and taste in the channel catfish : an electrophysiological study of the responses to amino acids and derivatives. J. Comp. Physiol., $123: 357-371$.

CARR W.E.S., HALL R.E., GURIN S., 1974. Chemoreception and the role of proteins : a comparative study. Comp. Biochem. Physiol., 47 A : 559 - 566.

CARR W.E.S., CHANEY T.B., 1975. Cheminal stimulation of feeding behavior in the pinfish, Lagodon rhomboides: characterization and identification of stimulating substances extracted from shrimp. Comp. Biochem. Physiol., 54 A : $437-441$.

CARR W.E.S., GURIN S., 1975. Chemoreception in the shrimp, Palaemonetes pugio : comparative study of stimulatory substances in human serum. Biol. Bull. Mar. Biol. Lab. Woods Hole, $148: 380=392$.

CARR W.E.S., 1976. Chemoreception and feeding behavior in the pigfish, Orthopristis chrysopterus : characterization and identification of stimulatory substances in a shrimp extract. Comp. Biochem. Physiol., 55 A : $153-157$.

FUJIYA M., BARDACH J.E., 1966. A comparison between the external taste sense of marine and freshwater fishes. Bull. Jap. Soc. Scient. Fish., $32 \cdot 45-56$.

GOH Y., TAMURA T., 1978. The electrical responses of the olfactory tract to amino acids in Carp. Bull. Jap. Soc. Sci. Fish., 44 (4) : 341 - 344.

HARA T.J., LAW Y.M.C., HOBDEN B.R., 1973. Comparison of the olfactory response to amino acids in rainbow trout, brook trout and whitefish. Comp. Biochem. Physiol., $45 \mathrm{~A}: 969-977$.

HARA T.J., 1976. Structure activity relationships of amino acids in fish olfaction. Comp. Biochem. Physiol., 54 A : 31 - 36.

HARA T.J., 1976. Effects on $\mathrm{pH}$ on the olfactory responses to amino acids in rainbow trout, Salmo gairdneri. Comp. Biochem. Physiol., 54 A : 37 - 39.

HARA T.J., 1977. Further studies on the structure activity relationships of amino-acids in fish olfaction. Comp. Biochem. Physiol., 56 A : 559 - 565.

HASHIMOTO Y., KONOSU S., FUSETANI N., NOSE T., 1968. Attractants for eels in the extract of short-necked clams. I - Survey of constituents eliciting feeding behavior by the omission test. Bull. Jap. Soc. Sci., $34: 78-83$.

HOBSON E.S., 1963. Feeding behaviour in three species of sharks. Pac. Sci., $17: 171-194$.

HODGSON E.S., MATHEWSON R.F., GILBERT P.W., 1967. Electroencephalographic studies of chemoreception in sharks. In : P.W. GILBERT, R.F. MATHEWSON and D.P. RALL, eds. Sharks, skates and rays. Johns Hopkins, Baltimore, Md.

KEENLEYSIDE M.H.A., 1979. Diversity and adaptation in fish behaviour. Zoophysiology, vol. 11 : 208 p. E.d. HOAR, HOELLDOBLER, JOHANSEN, LANGER and LINDAUER, Springer-verlag. Berlin Heidelberg New York.

KLEEREKOPER H., MOGENSEN J., 1963. Role of olfaction in the orientation of Petromyzon marinus. I - Response to a single amine in prey's body odour. Physiol. Zool. 36 : $347-360$.

KLEEREKOPER H., 1967. Some effects of olfactory stimulation on locomotor patterns in fish. In Olfaction and taste (Proc. 2nd Internat. Symp. 1965). HAYASHI T. : $625-645$. Pergamon Press, Oxford.

LINDSTEDT K.J., 1971. Chemical control of feeding behavior. Comp. Biochem. Physiol., 39 A : $553-581$.

McBRIDE J.R. IDLER D.R., JONAS R.E.E., TOMLINSON N., 1962. Olfactory perception in juvenile salmon. I - Observations on response of juvenile sockeye to extracts of foods. 1. Fish. Res. Bd. Canada, 19 (2) : 327 - 334.

MATHEWSON R.F., HODGSON E.S., 1972. Klinotaxis and rheotaxis in orientation of sharks toward chemical stimuli. Comp. Biochem. Physiol., 42 A : $79-84$.

PAWSON M.G., 1977. Analysis of a natural chemical attractant for whiting Merlangius merlangus L. and Cod Gadus morhua L. using a behavioural bioassay. Comp. Biochem. Physiol., 56 A : 129 - 135.

STEVEN D.M., 1959. Studies on the shoaling behaviour of fish. 1 - Responses of two species to changes of illumination and to olfactory stimuli. J. Exp. Biol., $36: 261-280$. 
STRAHAN R., 1963 a. The behaviour of myxinoids. Acta Zool., $44: 73-102$.

STRAHAN R., 1963 b. The behaviour of Myxine and other myxinoids. In BRODAL A. FANGE R. (Eds), The biology of Myxine. Scand. Univ. Bookds, OSLO, 22 - 32.

SUTTERLIN A.M., SUTTERLIN N., 1971. Electrical responses of the olfactory epithelium of Atlantic salmon (Salmo salar). J. Fish. Res. Bd. Can., 28 : 565 - 572.

SUTTERLIN A.M., 1975. Chemical attraction of some marine fish in their natural habitat. $J$. Fish. Res. Bd. Can., 32 (6) : 729 - 738.

SUZUKI N., DON TUCKER, 1971. Amino acids as olfactory stimuli in freshwater catfish. Ictalurus catus (LINN.). Comp. Biochem. Physiol., 40 A : $399-404$.

TESTER A.L., 1963. The role of olfaction in shark predation. Fac. Sci., $17: 145-170$. 